\title{
ENTIRE VECTORS AND HOLOMORPHIC EXTENSION OF REPRESENTATIONS
}

\author{
BY \\ RICHARD PENNEY
}

\begin{abstract}
Let $G$ be a connected, simply connected real Lie group and let $U$ be a representation of $G$ in a complete, locally convex, topological vector space $\supset$. If $G$ is solvable, it can be canonically embedded in its complexification $G_{c}$. A vector $v \in \supset$ is said to be entire for $U$ if the map $g \rightarrow U_{g} v$ of $G$ into $\supset$ is holomorphically extendible to $G_{c}$. The space of entire vectors is an invariant subspace of the space of analytic vectors. $U$ is said to be holomorphically extendible iff the space of entire vectors is dense.

In this paper we consider the question of existence of holomorphic extensions We prove

Theorem. A unitary representation $U$ is holomorphically extendible to $G_{c}$ iff $G$ modulo the kernel of $U$ is type $R$ in the sense of Auslander-Moore [1].

In the process of proving the above results, we develop several interesting characterizations of entire vectors which generalize work of Goodman for solvable Lie groups and we prove a conjecture of Nelson concerning the relationship between infinitesimal representations of Lie algebras and representations of the corresponding Lie groups.
\end{abstract}

Introduction. Let $G$ be a connected, simply connected (c.s.c.) real solvable Lie group with Lie algebra $\mathcal{L}$. Let $\mathcal{L}_{c}$ denote the complexification of $\mathcal{L}$ and let $G_{c}$ be the corresponding c.s.c. complex Lie group. From the solvability of $G, G$ is canonically imbeddable as a closed subgroup of $G_{c}$. If $f: G \rightarrow \square$ is a mapping of $G$ into a complete locally convex topological vector space $\square$, we will say that $f$ is holomorphically extendible to $G_{c}$ if there is a continuous mapping $f_{c}: G_{c} \rightarrow \square$ which extends $f$ such that the map $z \rightarrow\left\langle f_{c}(z), w\right\rangle$ is a holomorphic map of $G_{c}$ into $C$ for all $w \in \beth^{\prime}$ (the space of continuous linear maps of $\supset$ into $C$ ). It can be shown that in this case the $\operatorname{map} f_{c}$ is a differentiable map of $G_{c}$ into $\supset$ in the sense that $\lim _{t \rightarrow 0}(f(z \exp t X)-f(z)) / t$ exists for all $X \in \mathcal{L}_{c}$ and $z \in G_{c}$ and defines a continuous function of $z$. (See $\S I I I .8$ of Grothendieck [5] for details.)

If $U$ is a strongly continuous representation of $G$ in $\supset$ by continuous operators, we define, following Goodman [2], $\mathcal{H}_{\infty}^{\omega}(U)$ (which we also denote by $\mathcal{H}_{\infty}^{\omega}$ when sufficiently clear) to be the space of vectors $v \in \beth$ for which the map $g \rightarrow U_{g} v$ of $G$ into $\square$ is holomorphically extendible to $G_{c}$. $\mathcal{H}_{\infty}^{\omega}$ is an invariant subspace of

Presented to the Society, January 17, 1972 under the title Analytic extension of representatives; received by the editors June 15, 1972 and, in revised form, February 5, 1973.

AMS (MOS) subject classifications (1970). Primary 43A65, $22 \mathrm{E} 45$.

Key words and phrases. Analytic vector, type- $R$, Lie group, holomorphic extension. 
$\square$ and the restriction of $U$ to $\mathcal{H}_{\infty}^{\omega}$ is extendible to an analytic representation of $G_{c}$ in $\mathcal{H}_{\infty}^{\omega}$. When $\mathcal{H}_{\infty}^{\omega}$ is dense in $\square$ we shall say that $U$ has an analytic extension and we will call the extension of the part of $U$ in $\mathcal{H}_{\infty}^{\omega}$ the analytic extension of $U$. We shall denote it by $U^{c}$ or simply $U$ when clear.

In this paper we are interested in obtaining conditions under which the analytic extensions exist. The structure of the paper is as follows:

In §I we develop several infinitesimal characterizations of entire vectors which generalize results of Goodman [3] in the nilpotent-unitary case and of Nelson [8] in the analytic vector case. We also remark that there is a simple proof of a conjecture of Nelson concerning the relationship of infinitesimal representations of Lie algebras and representations of the corresponding Lie groups.

In §II, we give a complete characterization of those unitary representations which have analytic extensions. We also obtain sufficient conditions for a general representation to have entire vectors. These conditions imply, in particular, that every Banach space representation of a type $R$ solvable Lie group has a dense set of entire vectors. Our methods are analogous to those used by Robert Moore for analytic vectors [7].

0 . Notation. Throughout the rest of this paper we shall assume that the notation established above (e.g., $G, \sqsupset, \beth^{\prime}, \mathcal{L}$, etc.) remains in force. In addition, if $K$ is a Lie group, $\mathcal{L}(K)$ will denote its Lie algebra.

$N$ will denote the natural numbers.

If $a_{1}, \ldots, a_{p}$ are elements of some associative algebra and $\left(n_{1}, \ldots, n_{p}\right)=n$ $\in N^{p}$, then $\left(a_{1}, \ldots, a_{p}\right)^{n}=a_{1}^{n_{1}} \cdots a_{p}^{n_{p}}$. We adopt the usual conventions concerning multi-indices-e.g. $n !=n_{1} ! \cdots n_{p} !,|n|=\sum n_{i}$, etc.

We define $C^{n}(U)$ to be the space of vectors $v$ in $\square$ such that $g \rightarrow U(g) v$ is a $C^{n}$ map of $G$ into $\supset$ and $C^{\infty}(U)=\bigcap_{n=1}^{\infty} C^{n}(U)$. For $v \in C^{1}(U)$, and $X \in \mathcal{L}$, define $\partial U(X) v=\lim _{t \rightarrow 0}(U(\exp t X) v-v) / t$.

If $X=\left(X_{1}, \ldots, X_{n}\right) \in \mathcal{L} \times \mathcal{L} \times \cdots \times \mathcal{L}$, we set $\partial U(X)=\left(\partial U\left(X_{1}\right), \ldots\right.$, $\left.\partial U\left(X_{n}\right)\right)$.

I. Infinitesimal characterizations. Let $X=\left(X_{1}, \ldots, X_{d}\right)$ be an ordered Jordan Hölder basis for $\mathcal{L}$. (Recall that an ordered basis $\left(X_{1}, \ldots, X_{d}\right)$ is an ordered Jordan Hölder basis for $\mathcal{L}$ if $\left[X_{i}, X_{i+1}\right] \equiv 0 \bmod \left\{X_{i+1}, \cdots, X_{n}\right\}$. Every solvable Lie algebra has such a basis.)

We have the following characterization of entire vectors.

Proposition. (1.1). $v \in C^{\infty}(U)$ is an entire vector for $U$ iff the family of sets $S_{v, t}=\left\{(U(X) / n !) t^{|n|} \mid n \in N^{d}\right\}$ is bounded for all $t>0$.

Proof. Since $\left\{X_{1}, i X_{1}, X_{2}, i X_{2}, \ldots, X_{d}, i X_{d}\right\}$ is a Jordan Hölder basis for $\mathcal{L}_{c}$, the $\operatorname{map}\left(z_{1}, \ldots, z_{d}\right) \rightarrow \exp z_{1} X_{1} \cdots \exp z_{d} X_{d}$ of $C^{d}$ into $G_{c}$ provides global holomorphic coordinates for $G_{c}$.

If $v$ is entire for $U$ and if $w \in \beth^{\prime}$, it follows from the Cauchy estimates for $\left(z_{1}, \ldots, z_{d}\right) \rightarrow\left\langle U\left(\exp z_{1} X_{1} \cdots \exp z_{d} X_{d}\right) v, w\right\rangle$ that $\left\langle S_{v, l}, w\right\rangle$ is bounded for all $t>0$. Thus, $S_{v, t}$ is weakly, and hence strongly, bounded. 
Conversely, suppose $S_{v, t}$ is bounded for all $t>0$. Consider, for $z \in C^{d}$, the series

$$
\phi(z)=\sum_{n \in N} \frac{z^{n}}{n !} \partial U(X)^{n} v .
$$

(Recall $X=\left(X_{1}, \ldots, X_{d}\right)$.) By standard power series estimates, $\phi(z)$ is Cauchy in any continuous seminorm on $\square$ and hence converges in $\square$ and defines a holomorphic map of $C^{d}$ into $\supset$. We claim that for $t=\left(t_{1}, \ldots, t_{d}\right) \in \mathbf{R}^{d}$,

$$
\phi\left(t_{1}, \ldots, t_{d}\right)=U\left(\exp t_{1} X_{1}\right) U\left(\exp t_{2} X_{2}\right) \cdots U\left(\exp t_{d} X_{d}\right) v .
$$

If $t_{2}=t_{3}=\cdots=t_{d}=0$, this follows from Taylor's theorem, for let $w \in \beth^{\prime}$. Then

$$
\begin{aligned}
& \left\langle U\left(\exp t_{1} X_{1}\right) v, w\right\rangle-\sum_{0}^{K}\left\langle\frac{\partial U\left(X_{1}\right)}{n !} v t_{1}^{n}\right\rangle \\
& =\int_{0}^{t_{1}}\left\langle U\left(\exp S X_{1}\right) \partial U\left(X_{1}\right)^{K+1} v, w\right\rangle \frac{\left(t_{1}-S\right)^{K}}{K !} d S .
\end{aligned}
$$

The integrand is bounded independently of $K$. In fact, if $u \in \beth^{\prime}$, the family of linear functionals $u \circ U\left(\exp S X_{1}\right)$ as $S$ ranges from 0 to $t_{1}$, is pointwise $\left(\sigma\left(E^{\prime}, E\right)\right)$ bounded and hence strongly $\left(\beta\left(E^{\prime}, E\right)\right)$ bounded. Thus if $B$ is any bounded set, $\cup_{0 \leq s \leq t} U(\exp S X) B$ is weakly, and hence strongly, bounded. Applying this reasoning with $B=\left\{\partial U\left(X_{1}\right)^{n} / n !\left(2 t_{1}\right)^{n} v \mid n \in N\right\}$, we see

$$
\begin{aligned}
\left|\int_{0}^{t_{1}}\left\langle U(\exp S X) \partial U\left(X_{1}\right)^{K+1} v, w\right\rangle \frac{\left(t_{1}-S\right)^{K}}{K !} d S\right| \\
\leq M \frac{K+1}{\left(2 t_{1}\right)^{K+1}} \int_{0}^{t_{1}}\left(t_{1}-S\right)^{K} d S=M 2^{-(K+1) .}
\end{aligned}
$$

Thus (1) above converges to zero and $\phi\left(t_{1}, 0, \ldots, 0\right)=U\left(\exp t_{1} X_{1}\right) v$. The same reasoning applies to the vector $\partial U\left(X_{2}\right)^{n_{2}} \cdots \partial U\left(X_{d}\right)^{n_{d}} v$ ( $n_{i}$ fixed). Thus if $\bar{X}$ $=\left(X_{2}, \ldots, X_{d}\right)$ and $t=\left(t_{1}, \ldots, t_{d}\right) \in R^{d}$, then

$$
\begin{aligned}
\langle\phi(t), w\rangle & =\sum_{n \in N^{d}} \frac{t^{n}}{n !}\left\langle\partial U(X)^{n} v, w\right\rangle \\
& =\sum_{\bar{n} \in N^{d-1}} \frac{t^{\bar{n}}}{\bar{n} !} \sum_{n=0}^{\infty} \frac{t_{1}^{n}}{n !}\left\langle\partial U\left(X_{1}\right)^{n} \partial U(X)^{\bar{n}} v, w\right\rangle \\
& =\sum_{n \in N^{d-1}} \frac{t^{\bar{n}}}{(\bar{n}) !}\left\langle U\left(\exp t_{1} X_{1}\right) \partial U(\bar{X})^{n} v, w\right\rangle \\
& =\sum_{n \in N^{\alpha-1}} \frac{t^{n}}{(\bar{n}) !}\left\langle\partial U(X)^{n} v, U^{\prime}\left(\exp t X_{1}\right)(w)\right\rangle
\end{aligned}
$$

where ' denotes adjoint. Continuing by induction we see that

$$
\langle\phi(t), w\rangle=\left\langle U\left(\exp t_{1} X\right) U\left(\exp t_{2} X_{2}\right) \cdots U\left(\exp t_{d} X_{d}\right) v, w\right\rangle,
$$


i.e.

$$
\phi(t)=U\left(\exp t_{1} X_{1} \cdots \exp t_{d} X_{d}\right) v \text {. Q.E.D. }
$$

Remark. In the above proof we used only that the "matrix elements" $g \rightarrow\langle U(g) v, w\rangle$ were holomorphically extendible to $G_{c}$. Also, we concluded that $g \rightarrow U(g) v$ was expandible, in terms of our global coordinates, in a convergent power series about 0 . Hence we have

Corollary (1.2). The following are equivalent:

(a) $v$ is entire;

(b) $g \rightarrow\left\langle U_{g} v, w\right\rangle$ is holomorphically extendible to $G_{c}$ for all $w \in \Xi^{\prime}$;

(c) $g \rightarrow U_{g} v$ is expandible in terms of global coordinates in an absolutely convergent power series.

Entire vectors satisfy a seemingly stronger property than that of (I.1).

Theorem (I.3).Let $\mathcal{L}$ be a finite subset of $\mathcal{L}$. If $v \in \mathcal{H}_{\infty}^{\omega}$, then for all $t>0$ the set

$$
\mathcal{R}_{v, t}=\left\{\frac{A_{1} \cdots A_{K}}{K !} v t{ }^{K} \mid A_{i}=\partial U\left(R_{i}\right), R_{i} \in \mathcal{R}, K \in N\right\}
$$

is bounded in $\square$.

The proof is a counting argument utilizing the commutators of the $A_{i}$. The main algebraic step is contained in the following lemma.

Let $\mathcal{A}$ be an associative algebra. If $\chi x$ and $y$ are subsets of $A$, let $x^{n}$ denote the set of products of $n$ elements from $\chi$ and let $(X, Y)^{n, m}$ denote the set of products of $n+m$ elements from $X \cup Y, n$ of which come from $X$ and $m$ of which come from $y$. For $y \in A$, let ad $y: A \rightarrow A$ be the map ad $y(x)=x y-y x$.

Lemma 1. If $\mathcal{X}$ and $Y$ are subsets of $\mathcal{A}$, then every $a \in(X, Y)^{n, m}$ is expressible in the form $a=\sum_{K=0}^{m} a_{K}$ where each $a_{K}$ is a sum of at most $n^{K}\left(\begin{array}{c}m \\ K\end{array}\right)$ terms of the form

$$
\begin{array}{r}
y_{1}^{\prime} \cdots y_{m-K}^{\prime} \cdot\left[\operatorname{ad} y_{1} \cdots \operatorname{ad} y_{K_{1}}\left(x_{1}\right)\right]\left[\operatorname{ad} y_{K_{1}+1} \cdots \operatorname{ad} y_{K_{2}}\left(x_{2}\right)\right] \\
\cdots\left[\operatorname{ad} y_{K_{n-1}+1} \cdots \operatorname{ad} y_{K}\left(x_{n}\right)\right]
\end{array}
$$

where $y_{i}^{\prime}$ and $y_{i} \in \mathcal{Y}, x_{i} \in \mathcal{X}$ and where $0 \leq K_{1} \leq K_{2} \leq \cdots \leq K_{n-1} \leq K$. (If $K_{j}=K_{j+1}$, then we take ad $y_{K_{j}+1} \cdots$ ad $y_{K_{j+1}}\left(x_{j+1}\right)=x_{j+1}$. We treat the cases $K_{1}=0$ and $K_{n-1}=K$ similarly.)

Proof. Let $Z(n, m, K)$ be the set of terms of the form of (2) above union the set $\{0\}$. The lemma asserts that $(X, Y)^{n, m} \subset \sum_{K=0}^{m} m^{K}\left(\begin{array}{c}m \\ K\end{array}\right) Z(n, m, K)$. The relations $Z(n, 0,0)=X^{n}$ and $Z(0, m, 0)=Y^{m}$ imply that the lemma is true if either $m=0$ or $n=0$. Assume that the lemma is false and let $(n, m)$ be the smallest pair of integers for which it is false relative to the ordering $(a, b) \leq(c, d)$ iff $a \leq c$ and $b \leq d$. Note that $n>0$ and $m>0$. Let $a \in(X, Y)^{n, m}$. Either 
$a=a^{\prime} x$ where $x \in \mathcal{X}$ and $a^{\prime} \in(X, Y)^{n-1, m}$ or $a=a^{\prime} y$ where $a^{\prime} \in(X, Y)^{n, m-1}$ and $y \in \mathcal{Y}$. In the first case, the relation $Z(n-1, m, K) \cdot \chi \subset Z(n, m, K)$ and the inductive hypothesis imply that the lemma is true for $a$. In the second case, the relation $Z(n, m-1, K) \cdot y \subset n Z(n, m, K+1)+Z(n, m, K)$ (which follows easily from the identity $v_{1} v_{2} \cdots v_{n} \cdot y=\sum_{j=1}^{n} v_{1} \cdots$ ad $\left.y\left(v_{j}\right) \cdots v_{n}+y v_{1} \cdots v_{n}\right)$ and the inductive hypothesis imply that

$$
a \in \sum_{K=0}^{m-1} n^{K}\left(\begin{array}{c}
m-1 \\
K
\end{array}\right) n Z(n, m, K+1)+\sum_{K=0}^{m-1} n^{K}\left(\begin{array}{c}
m-1 \\
K
\end{array}\right) Z(n, m, K) .
$$

Regrouping and using the identity

$$
\left(\begin{array}{c}
m-1 \\
K-1
\end{array}\right)+\left(\begin{array}{c}
m-1 \\
K
\end{array}\right)=\left(\begin{array}{l}
m \\
K
\end{array}\right)
$$

we see that the lemma is true for $a$. This contradiction proves the lemma. Q.E.D.

For the next lemma, let $C$ be a vector space and let $\mu$ be a Minkowski functional on $C$ corresponding to a balanced but not necessarily absorbing convex set (i.e. $\mu$ satisfies $\mu(x+y) \leq \mu(x)+\mu(y)$ and $\mu(\alpha y)=|\alpha| \mu(y)$ but $\mu$ may assume infinite values). Let $\mathcal{A}$ be an algebra of linear operators on $C$ and let $\mathcal{N}$ be a finite dimensional vector subspace of $A$ with ordered basis $X=\left(x_{1}, \ldots\right.$, $\left.x_{d}\right)$. Let $\mathcal{Y}$ be a finite subset of $\mathcal{A}$ for which ad $y$ leaves $\mathcal{N}$ invariant for $y \in \mathcal{Y}$. Then

Lemma 2. If $v \in C$ is such that

$$
\left\{\frac{y}{m !} \cdot \frac{x}{n !}(v) t^{n+m} \mid x \in \mathcal{X}^{n}, y \in \mathcal{Y}^{m}, n, m \in N\right\}
$$

is bounded in $\mu$ for all $t>0$, then $\left\{(a / K !)(v) t^{K} \mid a \in(\chi \cup Y)^{K}, K \in N\right\}$ is bounded in $\mu$ for all $t>0$.

Proof. Let $a \in(\chi \cup \mathcal{Y})^{j}$. Then $a \in(\chi, Y)^{n_{m} m}$ for some $n, m \in N, n+m$ $=j$. Write $a=\sum_{K=0}^{m} a_{K}$ where the $a_{K}$ are as in Lemma 1. We estimate $\mu\left(a_{K}(v)\right)$ by estimating terms of the form of (2) above. In particular, following Goodman [2], we put on $\mathcal{N}$ the norm $\left|\sum_{i=1}^{d} c_{i} x_{i}\right|=\sum_{i=1}^{d}\left|c_{i}\right|$. Since $\mathcal{N}$ is finite dimensional, ad $y, y \in \mathcal{Y}$,defines a bounded linear transform (with respect to $|\cdot|$ ) of $\mathcal{N}$ into itself. Let $M$ be a common bound for these transforms.

Also, it is easily shown that if $n_{1}, \ldots, n_{q} \in \mathcal{N}$ and $a \in \mathcal{A}$, then

$$
\mu\left(a n_{1} \cdots n_{q}(v)\right) \leq\left|n_{1}\right| \cdots\left|n_{q}\right| \sup _{x \in X^{q}} \mu(a x(v)) .
$$

Thus, if $b$ is any term of the form of (2) above, $t \geq r>0$ and $C_{t}$ is a bound on the set (3) above, then 


$$
\begin{aligned}
\mu(b(v)) & \leq M^{K} \sup _{x \in X^{n}} \mu\left(y_{1}^{\prime} \cdots y_{m-K}^{\prime} x(v)\right) \\
& \leq M^{K} \sup _{y \in Y^{m-K}, x \in X^{n}} \mu\left(\frac{y}{(m-K) !} \frac{x}{n !}(v) t^{n+(m-K)}\right) \cdot t^{-(n+(m-K))} n !(m-K) ! \\
& \leq M^{K} C_{t} t^{-n} r^{-(m-K)} n !(m-K) ! .
\end{aligned}
$$

Thus,

$$
\begin{aligned}
\mu(a(v)) & \leq \sum_{K=0}^{m} \mu\left(a_{K}(v)\right) \\
& \leq \sum_{K=0}^{m} n^{K}\left(\begin{array}{c}
m \\
K
\end{array}\right) M^{K} C_{t} t^{-n} r^{-(m-K)} n !(m-K) ! \\
& \leq C_{t} t^{-n} r^{-m} n ! m ! \sum_{K=0}^{\infty} \frac{(n M r)^{K}}{K !} \\
& =C_{t}\left(t e^{-M r}\right)^{-n} r^{-m} n ! m ! \\
& \leq C_{t}\left(e^{M r} / t+1 / r\right)^{n+m} 2^{n+m} j ! \\
& =C_{t}\left(\frac{2 r t}{r e^{M r}+t}\right)^{-j} j ! .
\end{aligned}
$$

This implies the lemma since $2 r t /\left(r e^{M r}+t\right)$ can be made arbitrarily large.

To prove (I.3) let $\rho$ be a Minkowski functional based on a not necessarily absorbing convex set in $\beth$. We shall show that if the $S_{v, l}$ of (I.1) are bounded in $\rho$ for all $t>0$, then the $\mathcal{R}_{v, t}$ are also bounded in $\rho$. If $\operatorname{dim} G=1$, this is clear, so suppose $d=\operatorname{dim} G>1$ and the above assertion is true for all groups of lower dimensions and all such $\rho$.

For $s>0$ and $w \in C^{\infty}(U)$, define

$$
\mu_{s}(w)=\sup _{n \in N} \rho\left(\frac{\partial U\left(X_{1}\right)^{n}}{n !} w s^{n}\right) .
$$

$\mu_{s}$ is a Minkowski functional and, letting $\bar{X}=\left(X_{2}, \ldots, X_{d}\right),\left\{\left(\partial U(\bar{X})^{n} / n !\right) v t|n| \mid n\right.$ $\left.\in N^{d-1}\right\}$ is bounded in $\mu_{s}$. Since $\left(X_{1}, \ldots, X_{d}\right)$ is a Jordan Hölder basis, $\operatorname{span}\left\{X_{2}, \ldots, X_{d}\right\}$ is an ideal in $\mathcal{L}$. It follows then from the inductive hypothesis that if $X=\left\{\partial U\left(X_{2}\right), \ldots, \partial U\left(X_{d}\right)\right\}$, then $\left\{\mu_{s}\left((A(v) / n !) t^{n}\right) \mid A \in X^{n}, n \in N\right\}$ is bounded-i.e.

$$
\left\{\frac{\partial U\left(X_{1}\right)^{n_{1}}}{n_{1} !} \frac{A(v)}{n !} s^{n_{1}} t^{n} \mid n_{1}, n \in N, A \in X^{n}\right\}
$$

is $\rho$ bounded. It now follows from Lemma 2 that (I.2) is true with $\mathcal{R}$ $=\left\{X_{1}, \ldots, X_{d}\right\}$. (I.2) for general $\mathcal{C}$ follows easily from this. Q.E.D.

As corollaries to the above proof we have the following facts, all due to Goodman in the nilpotent case. Our proofs are inspired by Goodman's. 
Corollary (I.4.) Let $U$ be a unitary representation of $G$ in a Hilbert space \&. If $G$ is a product of two analytic subgroups $K$ and $N$ with $N$ normal then $v$ is entire for $U$ if (and only if) $v$ is entire for $\left.U\right|_{N}$ and $\left.U\right|_{K}$.

Proof. Let $Y$ and $X$ be, respectively, bases for $\mathcal{L}(K)$ and $\mathcal{L}(N)$. Let $Y=\partial U(Y)$ and $X=\partial U(X)$, considered as sets of operators on $C=C^{\infty}(U)$. Let $A$ be the algebra they generate and let $\mu$ be the seminorm $|(\cdot, v)|$. If $y \in Y^{n}$ and $x \in X^{m}$, $n, m \in \mathcal{N}$, then from the unitarity of $U, y^{*} \in Y^{n}$ and

$$
\mu\left(\frac{y}{n !} \frac{x}{m !}(v) t^{n+m}\right)=\left|\left(\frac{x}{m !}(v) t^{m}, \frac{y^{*}}{n !}(v) t^{n}\right)\right| \leq\left\|\frac{x}{m !}(v) t^{m}\right\|\left\|\frac{y^{*}}{n !}(v) t^{n}\right\| .
$$

By (I.3) this is bounded independently of $m$ and $n$. Thus, by Lemma 2 above, for all $t>0$ there is a constant $C_{t}>0$ such that if $a \in(X \cup Y)^{K}$, then $\mu(a(v))$ $\leq C_{t} K ! t^{-K}$. Hence, if $a \in(\mathcal{X} \cup Y)^{K}$, then

$$
t^{-2 K}(2 K) ! C_{t} \geq \mu\left(a^{*} a(v)\right)=\|a(v)\|^{2} .
$$

The entirety of $v$ follows from (I.2). Q.E.D.

Corollary (1.5). If $U$ is as in Corollary (I.4) then $v$ is entire for $U$ iff $v$ is entire for each of the representations $t \rightarrow U\left(\exp t X_{i}\right), i=1, \ldots, d$, where $\left(X_{1}, \ldots, X_{d}\right)$ is an ordered Jordan Hölder basis.

Proof. Follows by induction from Corollary (I.4).

Corollary (1.6). If $U$ is as in Corollary (1.4), then $v$ is entire for $U$ iff $g \rightarrow\left(U_{g} v, v\right)$ is holomorphically extendible to $G_{c}$.

Proof. Reasoning as in the "only if" part of (I.1), it is easily seen that for $X \in \mathcal{L}, t>0,\left\{\left(\partial U(X)^{n} / n !\right)(v) t^{n} \mid n \in N\right\}$ is bounded in the seminorm $|(\cdot, v)|$. Hence, as in the proof of Corollary (I.5), $v$ is entire for $t \rightarrow U(\exp t X)$ from (I.1). Since $X$ was arbitrary, the conclusion follows from Corollary (I.5). Q.E.D.

Remark (I.7). The conclusion of (I.3), since it requires neither the simpleconnectedness nor solvability of $G$, is usually taken as the definition of entirety of $v$. We shall henceforth assume (I.3) as the definition of entirety. Note, however, that by lifting to the universal covering group, one may assume simpleconnectedness without loss of generality.

Note, also, that (I.3) establishes that entire vectors are a type of analytic vector (see Nelson [8]). In fact, if in (I.3), $\mathcal{Q}=\left\{X_{1}, \ldots, X_{d}\right\}$ is any basis of $\mathcal{L}$, then the analytic vectors for $U$ are the set of $v$ such that $\angle R_{v, t}$ is bounded for some $t>0$. As Nelson noted, this definition depends only on the representation of $\mathcal{L}$ given by $X \rightarrow \partial U(X)$ and not on $U$.

In [8], Nelson showed that a representation $\rho$ of a Lie algebra \& by a family of partially defined skew symmetric operators in a Hilbert space of having a common, dense, invariant domain is the restriction of the differential of a unitary representation of the corresponding c.s.c. Lie group $G_{0}$ iff there is a $t>0$ such 
that the space of vectors $v$ for which $\mathcal{R}_{v, t}$ is bounded (denoted $\mathcal{L}_{t}$ ) is dense in $\mathcal{H}_{\text {. }}$ Nelson remarked that it was unknown if it is sufficient to assume only that the space of analytic vectors is dense. We remark that it is indeed sufficient.

In fact, let $X=\left(X_{1}, \ldots, X_{d}\right)$ be a basis for (B) and let $\Omega \subset R^{d}$ be an open neighborhood of 0 for which the map $\phi\left(t_{1}, \ldots, t_{d}\right) \rightarrow \exp t_{1} X_{1} \cdots \exp t_{d} X_{d}$ is a homeomorphism of $\Omega$ onto an open subset $\Omega^{\prime}$ in $G_{0}$. In [8] Nelson showed that under the assumption of density of the analytic vectors the $\rho(Y)$ are essentially skew-adjoint for $Y \in$ BS. Hence for $t \in R$ we may form the operator exp $t \overline{\rho(Y)}$ which is unitary by Stone's theorem, and hence we may define a mapping $U$ of $\Omega^{\prime}$ into unitary operators via

$$
\exp t_{1} X_{1} \cdots \exp t_{d} X_{d} \rightarrow \exp t_{1} \overline{\rho\left(X_{1}\right)} \cdots \exp t_{d} \overline{\rho\left(X_{d}\right)}
$$

for $t=\left(t_{1}, \ldots, t_{d}\right) \in \Omega$. If $U$ can be shown to be a local representation of $\Omega^{\prime}$, it will follow as in Nelson that $U$ is extendible to a unitary representation of $G_{0}$ whose differential extends $\rho$. Now let $v$ be an analytic vector for $\rho$, say $v \in \mathcal{H}_{\text {. }}$. It is easily seen that $C_{v}=\operatorname{span}\left\{\rho\left(Y_{1}\right) \cdots \rho\left(Y_{K}\right) v \mid Y_{i} \in \mathcal{Q}, K \in N\right\}$ is a $\rho$ invariant subspace of $\alpha_{s}$ for any $s<t$. Let $\alpha_{v}=\bar{C}_{v}$ and let $\tilde{\rho}$ be the representation of (c) defined by $\tilde{\rho}(X)=\left.\rho(X)\right|_{c}$ considered as a partially defined operation on $\alpha_{v}$. By Nelson's theorem the map

$$
\exp t_{1} X_{1}, \ldots, \exp t_{d} X_{d} \rightarrow \exp t_{1} \overline{\tilde{\rho}\left(X_{1}\right)} \cdots \exp t_{d} \overline{\tilde{\rho}\left(X_{d}\right)}
$$

for $t=\left(t_{1}, \ldots, t_{d}\right) \in \Omega$ defines a local representation $U$ of $\Omega^{\prime}$ in $\alpha_{v}$. Hence, to prove that $U$ is a local representation, it suffices to show that the restriction of $U$ to $\alpha_{v}$ is equal to $\tilde{U}$ for all $v \in \mathcal{\alpha}_{v}$. But, if $w \in C_{v}$ it can be shown via the reasoning of Theorem (I.1) that $\tilde{U}(\phi(t)) w$ and $U(\phi(t)) w$ are both given by the same power series for $t$ close enough to zero (independently of $w$ ). Since $C_{v}$ is dense in $d_{v}$, our remark is proven.

It should be noted that it now follows from the converse of Nelson's theorem that $\alpha_{t}$ is in fact dense for some $t>0$.

Example (1.8). Let $G^{A}$ be the two dimensional Lie group with Lie algebra spanned by $X$ and $Y$ satisfying $[X, Y]=\alpha_{0} Y$. It is well known that every infinite dimensional irreducible unitary representation of $G^{A}$ is realizable in $L^{2}(R)$ in such a way that $\partial U(X)=\partial / \partial t$ and $\partial U(Y)=i \lambda e^{\alpha_{0} t}, \lambda$ and $\alpha_{0}$ some real constant. We remark for the purposes of Example (I.9) that for every $\lambda$ and $\alpha_{0} \neq 0$ in $R$ there is such a representation. Goodman showed that no such representation could have nonzero entire vectors. From this, he was able to show, among other things, that no locally injective representation of $G^{A}$ could have entire vectors. See [2] for this.

Example (1.9). Let $\alpha=\alpha_{0}+\alpha_{1} i \in C$ and let $G^{\alpha}$ be the group structure on $C \times R$ defined by $(c, r) *\left(c^{\prime}, r^{\prime}\right)=\left(c+e^{\alpha r} c^{\prime}, r+r^{\prime}\right) . G^{\alpha}$ is a solvable, connected, simply connected Lie group. Let $\lambda$ be a nonzero complex number. Let $U^{\lambda}$ be the. representation of $G^{\alpha}$ in $L^{2}(R)$ given by 


$$
U_{(c, r)}^{\lambda} f(t)=\exp i \operatorname{Re}\left(\lambda e^{\alpha t} c\right) f(t+r) .
$$

It can be shown via Mackey's theory of induced representations that for $\operatorname{Re} \alpha \neq 0, U^{\lambda}$ is irreducible and every irreducible representation of $G^{\alpha}$ is of this form. The Lie algebra of $G^{\alpha}$ is isomorphic to $C \times{ }_{S} R$ with the commutation rule $\left[(c, r),\left(c^{\prime}, r^{\prime}\right)\right]=\left[\alpha\left(c r^{\prime}-r c^{\prime}, 0\right)\right]$. Let $Y_{1}=(i, 0), Y_{2}=(1,0)$ and $X=(0,1)$. Then

$$
\partial U\left(Y_{1}\right) f(t)=-i \operatorname{Im}\left(\lambda e^{\alpha t}\right) f(t), \quad \partial U\left(Y_{2}\right)=i \operatorname{Re}\left(\lambda e^{\alpha t}\right) f(t)
$$

and

$$
\partial U\left(X_{1}\right) f(t)=(\partial / \partial t) f(t)
$$

We claim $U^{\lambda}$ has no nonzero entire vectors. Suppose $f$ is entire for $U^{\lambda}$. Let

$$
Y=\sqrt{\partial U\left(Y_{1}\right)^{2}+\partial U\left(Y_{2}\right)^{2}}=i\left|\lambda e^{\alpha t}\right|=i|\lambda| e^{a_{0} t} .
$$

From the inequalities $(n !)^{2} \geq 2^{-n}(2 n)$ ! and $\left\|Y^{n} f\right\|^{2} \leq\|f\|\left\|Y^{2 n} f\right\|$, we see

$$
\begin{aligned}
\left(\left\|Y^{n} f\right\| \frac{t^{n}}{n !}\right)^{2} & \leq 2^{-n}\|f\|\left\|Y^{2 n} f\right\| \frac{(2 t)^{2 n}}{(2 n) !} \\
& \leq 2^{-n}\|f\| \sum_{K=0}^{n}\left(\begin{array}{l}
n \\
K
\end{array}\right)\left\|\partial U\left(Y_{1}\right)^{2 K} \partial U\left(Y_{2}\right)^{2(n-K)} f\right\| \frac{(2 t)^{2 n}}{(2 n) !} .
\end{aligned}
$$

Since $2^{-n} \sum_{K=0}^{n}\left(\begin{array}{l}n \\ k\end{array}\right)=1$, this is bounded by (I.3).

Also, by (I.1), $\left\{\left\|(\partial / \partial t)^{n} f\right\| t^{n} / n !\right\}$ is bounded for all $t>0$. Hence, by (I.5) and (I.1), $f$ is entire for the representation of $G^{A}$ considered in Example (I.8). Hence, by Goodman's result, $f=0$.

It follows from this, as in Goodman [2], that no locally injective representation of $G^{\alpha}, \operatorname{Re} \alpha \neq 0$, can have nonzero entire vectors.

If $\operatorname{Re} \alpha=0$, every unitary representation of $G^{\alpha}$ has a dense set of entire vectors. This will follow from the general theory of §II.

II. In this section $U$ will be a representation of $G$ in a Hilbert space \&. Recall that $G$ is said to be class $R$ iff the eigenvalues of ad $X$ for $X \in \mathcal{L}$ are all pure imaginary.

Proposition (II.1). If $U$ has a holomorphic extension, then $G$ modulo the kernel of $U$ is class $R$.

Proof. If $K$ is the connected component of the identity of the kernel of $U, U$ induces a representation of $G / K$ and clearly this representation has a holomorphic extension iff $U$ does. Therefore, we may assume that $U$ is locally injective. Then, by Examples (I.8) and (I.9), $G$ can contain no analytic subgroups isomorphic to $G^{\alpha}(\operatorname{Re} \alpha \neq 0)$ or $G^{A}$. This implies that $\mathcal{L}$ is class $R$ for if not, there is an $X \in \mathcal{L}$ and a $Y \in \mathcal{L}_{c}$ for which $[X, Y]=\alpha Y$ where $\alpha \in C$, 
$\operatorname{Re} \alpha \neq 0$. If $Y=Y_{1}+i Y_{2}$ and $\left[Y_{1}, Y_{2}\right]=0$, then $\left\{X, Y_{1}, Y_{2}\right\}$ span a Lie algebra isomorphic to that of $G^{\alpha}$, which is a contradiction. Hence $\left[Y_{1}, Y_{2}\right] \neq 0$. But then $\left[X,\left[Y_{1}, Y_{2}\right]\right]=\left[\left[X, Y_{1}\right], Y_{2}\right]+\left[Y_{1},\left[X, Y_{2}\right]\right]=2 \operatorname{Re} \alpha\left[Y_{1}, Y_{2}\right]$. Hence $\left\{X,\left[Y_{1}, Y_{2}\right]\right\}$ span a Lie algebra isomorphic to $G^{A}$ which is a contradiction. Q.E.D.

The converse of (II.1) is true, but the proof is more involved. We begin by considering the $L^{p}$ left regular representation of $G$. We will call entire vectors for this representation $L^{p}$ entire vectors. We will denote the $L^{2}$ left regular representation by $L^{G}$.

Recall that a real Lie algebra $\mathcal{L}$ is said to be semisimply split by a semidirect product decomposition $\mathcal{L}=\beth+\mathcal{N}(\mathcal{N}$ an ideal) provided $\mathcal{N}$ is nilpotent, $\Xi$ is abelian and ad $\supset \mid \mathcal{N}$ is a semisimple family of linear transforms (i.e. there is a basis of $\mathcal{N}_{c}$ consisting of eigenvectors of the operators ad $\supset \mid \mathcal{N}$. A semidirect product decomposition of $G, G=T \cdot N$, semisimply splits $G$ provided the corresponding decomposition of $\mathcal{L}$ semisimply splits $\mathcal{L}$.

Lemma 1. If $G$ is class $R$ and $G=T \cdot N$ is a semisimple splitting of $G$, then $\mathcal{H}_{\infty}^{\omega}\left(L^{G}\right)$ is dense in $L^{2}(G)$. In fact, if $f \in \mathcal{H}_{\infty}^{\omega}\left(L^{T}\right)$ and $g \in \mathcal{H}_{\infty}^{\omega}\left(L^{N}\right)$, then $f \otimes g \in \mathcal{H}_{\infty}^{\omega}\left(L^{G}\right)$ where $f \otimes g$ is the function $f \otimes g(t n)=f(t) g(n)$ for $t \in T$, $n \in N$.

Proof. From the solvability of $G, G_{c}=T_{c} \cdot N_{c}$. Let $f$ and $g$ be as above. Since for $s_{1}, t \in T$ and $s_{2}, n \in N$,

$$
L^{G}\left(s_{1} s_{2}\right) f \otimes g(t n)=\left(L^{T}\left(s_{1}\right) f\right) \otimes\left(L^{N}\left(t^{-1} s_{1} s_{2} s_{1}^{-1} t\right) g\right)(t n)
$$

we may formally define an analytic extension of $L^{G}$ at $f \otimes g$ via $L^{G}\left(z_{1} z_{2}\right) f \otimes g(t n)$ $=\left(L^{T}\left(z_{1}\right) f\right) \otimes L^{N}\left(t^{-1} z_{1} z_{2} z_{1}^{-1} t\right) g(t n)$ for $z_{1} \in T_{c}, z_{2} \in N_{c}$. We need to show that this remains in $L^{2}$. This follows from the fact that the adjoint action of $T$ on $N_{c}$ has relatively compact orbits, and hence $\left\|L^{N}\left(t^{-1} z_{1} z_{2} z_{1}^{-1} t\right) g\right\|_{2}$ is a uniformly bounded function of $t$. The relative compactness follows from the class $R$ assumption for if $X_{1}, \ldots, X_{n}$ is a basis of $N_{c}$ consisting of eigenvectors for ad $\supset \mid \mathcal{N} \mathcal{N}_{c}$, then, for $Y \in T, c_{i} \in C$,

$$
\exp Y \cdot \exp \left(c_{1} X_{1}+\cdots+c_{n} X_{n}\right) \cdot \exp -Y=\exp \left(c_{1} e^{i a_{n}} X_{1}+\cdots+c_{n} e^{i a_{n}} X_{n}\right),
$$

where ad $Y\left(X_{i}\right)=i \alpha_{i} X_{i}, \alpha_{i}$ real. The required analyticity follows by an application of Morera's theorem and the verifiable fact that orbits of compact sets of $N_{c}$ under the adjoint action of $T$ on $N_{c}$ are relatively compact.

Hence, to conclude our proof, we need only show that $T$ and $N$ have dense sets of $L^{2}$ entire vectors. However, since $T$ and $N$ are nilpotent, it follows from results of Goodman [3, Corollary (5.5)] that the $L^{2}$ left regular representation of $T$ and $N$ have dense sets of entire vectors. Q.E.D.

We are now in a position to prove the converse of (II.1).

Theorem (II.2) $U$ has a holomorphic extension iff $G$ modulo the kernel of $U$ is class $R$. 
Proof. If $f$ is any $L^{1}$ entire vector for $L^{G}$, then it follows by standard arguments that $w=\int_{G} f(g) U_{g} v d g$ is entire for $U$, the analytic extension being given by $U_{z} w=\int L_{z^{-1}}^{G} f(g) U_{g}(v) d g$. If $G$ has a dense set of such $f$, then the linear span of the set of such $w$ is dense, as can be seen by the usual annihilator argument. Hence, it suffices to show that $G$ has a dense set of $L$ entire vectors. Since the pointwise product of two $L^{2}$ entire vectors is an $L^{1}$ entire vector (there is an obvious analytic extension) it suffices to consider $L^{G}$.

To do so, we use the semisimple splitting of Auslander-Moore [1, p. 132]. In particular, in [1] it was shown that $G$ can be imbedded as a normal subgroup in a connected, simply connected solvable, Lie group $G_{0}$ which can be semisimply split by factors $T$ and $N$ ( $N$ normal). Furthermore $G_{0}=T \cdot G$ and this decomposition is semidirect. $G_{0}$ can be chosen to be class $R$ for if $\mathcal{L}_{0}$ is the Lie algebra of $G_{0}$, the collection $\exists$ of elements in $\mathcal{L}_{0}$ with pure imaginary eigenvalues is a subspace which contains $\left[\mathcal{L}_{0}, \mathcal{L}_{0}\right]$ and hence is a Lie algebra. It is easily shown that if $\square$ and $\mathcal{N}$ are the Lie algebras of $T$ and $N$, then $\Im=\Im \cap \square+\mathcal{N}$ is a semisimple splitting of $\Im$. Also $\Im=\Im \cap \Im+\mathcal{L}$. Hence, taking $G_{0}$ to be class $R$, we know, by Lemma $1, L^{G_{0}}$ has an $L^{2}$ dense set of entire vectors.

Now, since $G_{0}=T \cdot G$, Haar measure on $G_{0}$ decomposes into a product of Haar measure on $T$ and $G$, and $L^{2}\left(G_{0}\right)$ can be decomposed as a direct integral of $L^{2}(G)$ over $T$ with respect to Haar measure. If $f \in L^{2}\left(G_{0}\right)$ and $t \in T$, the $t$ th component of $f$ is the map of $G$ into $C, f_{t}(g)=f(t g)$. This decomposition decomposes $\left.L^{G_{0}}\right|_{G}$ as $\int \oplus L^{t} d T(t)$ where $L^{t}(g)=L^{G}\left(t^{-1} g t\right)$. By Goodman's direct integral theorem [3, Lemma 3.1] it follows that almost every $L^{\prime}$ must have dense sets of entire vectors. It is easily shown that any entire vector for $L^{t}$ is entire for $L^{G}$. In fact $\mathcal{H}_{\infty}^{\omega}\left(L^{t}\right)=\mathcal{H}_{\infty}^{\omega}\left(L^{G}\right)$ for all $t \in T$. Q.E.D.

Remark. The procedure for producing entire vectors described above can be used to produce entire vectors having certain regularity properties. For example, let \|\| be a left invariant Riemannian metric on $G_{0}$. Suppose $f$ and $g$ are as in Lemma 1. Suppose also that for all compact sets $\Omega$ in $T$ and $\chi$ in $N$, there are positive constants $B_{\Omega}, B^{\prime}, C_{\chi}, C^{\prime},\left(B^{\prime}\right.$ and $C^{\prime}$ independent of $\Omega$ and $\left.\chi\right)$ such that

$$
\left|L_{z_{1}}^{T} f(t)\right| \leq B_{\Omega} \exp \left(-B^{\prime}\|t\|^{2}\right) \text { and }\left|L_{z_{2}}^{N} g(n)\right| \leq C_{\chi} \exp \left(-C^{\prime}\|n\|^{2}\right)
$$

for $z_{1} \in \Omega, z_{2} \in \chi, n \in N, t \in T$.

Then, from the above proof, it is easily seen that if $\Lambda$ is a compact subset of $G_{0}$, then there are positive constants $A_{\Lambda}$ and $A^{\prime}\left(A^{\prime}\right.$ independent of $\left.\Lambda\right)$ such that

$$
\left|L_{z_{1} z_{2}} f \otimes g(t n)\right| \leq A_{\Lambda} \exp \left(-A^{\prime}\|t n\|^{2}\right) .
$$

In fact, if $\Omega$ and $\chi$ are the projections of $\Lambda$ in $T_{c}$ and $N_{c}$ respectively, and if $\chi_{0}$ is the closure of the orbit of $\Omega \chi \Omega^{-1}$ by $T$ in $N_{c}$ (which is compact by the above proof) then by (1) and (2) and the fact that $\|t n\| \leq\|t\|+\|n\|$

$$
\begin{aligned}
\left|L_{z_{1} z_{2}} f \otimes g(t n)\right| & \leq B_{\Omega}\left(\exp \left(-B^{\prime}\|t\|^{2}\right)\right) \cdot C_{\chi_{0}} \exp \left(-C^{\prime}\|n\|^{2}\right) \\
& \leq B_{\Omega} C_{\chi_{0}} \exp \left(-A^{\prime}\|t n\|^{2}\right)
\end{aligned}
$$


where $A^{\prime}=\frac{1}{4} \inf \left(B^{\prime}, C^{\prime}\right)$ and where $z_{1} z_{2} \in \Lambda, t \in T, n \in N$.

From the proof of (II.2), for a.e. $t \in T$, the map $x \rightarrow(f \otimes g)(t x)=(f \otimes g)$ $(x)$ is, when restricted to $G$, entire for $L^{t}$. Hence, from the decomposition of $L^{G_{0}}$ as a direct integral of the $L^{\prime}, t \in T$ (see (II.2)) we see that for $z \in G_{c}$,

$$
\begin{aligned}
L^{G}(z)\left((f \otimes g)_{t}\right) & =L^{t}\left(t z t^{-1}\right)\left((f \otimes g)_{t}\right) \\
& =\left[L^{G_{0}}\left(t z t^{-1}\right)(f \otimes g)\right]_{\ell}=L^{G_{0}}\left(z t^{-1}\right)(f \otimes g) .
\end{aligned}
$$

Thus, if $\Delta$ is a compact subset of $G_{c}$, then for $k \in G, z \in \Delta$,

$$
\begin{aligned}
\left|L^{G}(z)\left((f \otimes g)_{t}\right)(k)\right| & =\left|L^{G_{0}}\left(z t^{-1}\right)(f \otimes g)(k)\right| \\
& \leq A_{\Delta^{-1}-1} \exp \left(-A^{\prime}\|k\|^{2}\right) .
\end{aligned}
$$

Thus $(f \otimes g)$, satisfies an estimate similar to (2) above.

Entire vectors satisfying (2) always exist for nilpotent groups. In fact, let $N$ be any c.s.c. nilpotent group and let $\mathcal{N}=\mathcal{L}(N)$. Let $\left\{X_{j}\right\}, j=1, \ldots, d$, be an ordered Jordan Hölder basis for $\mathcal{N}$ considered as elements of $\mathcal{N}_{c}$. For $z \in C$, set $g_{j}(z)=\exp z X_{j} \in N_{c}$. For $P \in N^{d}, P=\left(P_{1}, \ldots, P_{d}\right)$, let $A_{P}$ be the space of entire functions $f$ on $G_{c}$ for which the function $F\left(z_{1}, \ldots, z_{d}\right)$ $=f\left(\exp z_{1} X_{1} \cdots \exp z_{d} X_{d}\right)$ satisfies the hypothesis of Theorem (5.1) of [3], i.e. there are positive constants $A, B$ and $C$ for which

$$
\left|F\left(z_{1}, \cdots, z_{d}\right)\right| \leqslant C \exp \left\{-A\left(\sum\left|\operatorname{Re} z_{j}\right|^{P_{j}}\right)+B\left(\sum\left|\operatorname{Im} z_{j}\right|^{P_{j}}\right)\right\} .
$$

Goodman showed that there is a positive constant $m$ such that if $P_{K+1}>P_{K} m$ for all $K$, then $f \in \mathcal{A}_{P}$ implies that there are positive constants $C_{1}, A_{1}, D, q$ such that

$$
f\left(g_{j}(w) g_{1}\left(t_{1}\right) \cdots g_{d}\left(t_{d}\right)\right) \leq C_{1} \exp \left\{-A_{1} \sum_{i=1}^{d}\left|t_{i}\right|^{P_{1}}+D|w|^{q}\right\}
$$

for all $t_{i} \in R, w \in C$.

An obvious modification of Goodman's proof shows that there are constants $A_{1}, B_{1}, C_{1}, D, q$ (independent of $w$ and $z_{i}$ ) such that

$$
\left|f\left(g_{j}(w) g_{1}\left(z_{1}\right) \cdots g_{d}\left(z_{d}\right)\right)\right| \leq C_{1} \exp \left\{-A_{1} \Sigma\left|\operatorname{Re} z_{j}\right|^{P_{j}}+B_{1} \Sigma\left|\operatorname{Im} z_{j}\right|^{P_{j}}+D|w|^{q}\right\}
$$

for $z_{i}, w \in C$.

It follows that $A_{p}$ is invariant under complex left translations and that for $w=g_{1}\left(w_{1}\right) \cdots g_{d}\left(w_{d}\right), w_{i} \in C$,

$$
\left|f\left(w g_{1}\left(z_{1}\right) \cdots g_{d}\left(z_{d}\right)\right)\right| \leq C \exp \left\{-A \sum\left|\operatorname{Re} z_{i}\right|^{P_{1}}+B \sum\left|\operatorname{Im} z_{i}\right|^{P_{1}}+D \Sigma\left|w_{i}\right|^{q}\right\} .
$$


If $P_{1} \geq 2$, it follows that the restriction of $f$ to $G$ satisfies (2) above. (Note that $\left.\left\|g_{1}(t) \cdots g_{d}\left(t_{d}\right)\right\| \leq\left\|g_{1}\left(t_{1}\right)\right\|+\cdots+\left\|g_{d}\left(t_{d}\right)\right\| \leq\left|t_{1}\right|+\cdots+\left|t_{d}\right|.\right) \quad$ Goodman showed that $A_{P}$ is a dense subset of $L^{2}(G)$.

Corollary (II.3). Suppose $U$ is as in the introduction and $G$ is class $R$. Then if $U$ has a dense set of vectors $v$ for which $g \rightarrow U_{g} v e^{M\left\|_{g}\right\|}$ is bounded for some $M<0$ (depending on $v$ ), then $U$ has a dense set of entire vectors.

Proof. This follows as in Moore [7, Theorem 5,Chapter 6], where instead of the family of functions $f(t, \cdot)$, we regularize with respect to entire vectors satisfying (2) above.

Corollary. Every representation of $G$ in a Banach space has a dense set of entire vectors.

Proof. Banach space representations are known to satisfy the hypothesis of the previous corollary with respect to every vector.

Remarks. The proof Moore's theorem cited above is somewhat more complicated than required in our case as we can prove existence of the required integral via a Riemannian sum since our representation is given as being continuous.

Our results on unitary representations admit a nice description in terms of the Green kernel. (After Leon Green who, we are told, has studied this kernel extensively.)

Definition. Let $\mathcal{L}$ be a Lie algebra. The Green kernel of $\mathcal{L}$ is the smallest ideal $\mathscr{K}$ such that $\mathcal{L} / \mathscr{K}$ is type $R$. If $G$ is the connected, simply connected Lie group corresponding to $\mathcal{L}$, then the Green kernel of $G$ is the analytic subgroup of $G$ corresponding to $\mathfrak{K}$.

Then (2.2) may be paraphrased as

Corollary (II.4). $A$ unitary representation $U$ of a solvable Lie group has a dense set of entire vectors iff $U$ is the identity on the Green kernel. In any event, the closure of the set of entire vectors is the space of vectors left invariant by the Green kernel.

This corollary solves completely the question of when unitary representations of solvable Lie groups have entire vectors. However, as noted in Remark (I.7), the concept of entire vector is definable for nonsolvable groups.

To consider the general problem, let $G$. be a not necessarily solvable connected, simply connected Lie group. By the Levi decomposition $G$ is a semidirect product of a semisimple group $T$ and a normal solvable group $S$. If $U$ is any locally injective unitary representation of $G$ which has a dense set of entire vectors, the restriction of $U$ to $S$ has a dense set of entire vectors. Hence $S$ is type $R$. Similarly, by a theorem of Goodman (Goodman [2, Theorem 8.1]), $T$ must be compact.

Conversely, suppose $G$ is a semidirect product of a compact semisimple group $T$ and a normal solvable type $R$ group $S$. Since finite dimensional representations have bounded differentials, every vector for such a representation is entire. 
Hence, by the Peter-Weyl theorem there is a dense set of $L^{2}$ entire vectors for $T$. If $f$ and $g$ are $L^{2}$ entire vectors for $T$ and $S$ respectively, it follows as in Lemma 1 above that $f \otimes g$ is entire for $L^{G} \mid S$. It follows from formula (1) that $f \otimes g$ is entire for $L^{G} \mid T$ also. Hence from Theorem (I.4) (note that the proof of this theorem was "infinitesimal" and hence did not depend on the solvability of the group), $f \otimes g$ is an $L^{2}$ entire vector for $G$. Thus $G$ has a dense set of $L^{2}$ entire vectors. It is not difficult to show from the Leibniz product theorem for derivatives that the pointwise product of $L^{2}$ entire vectors is an $L^{1}$ entire vector. Finally, if $U$ is a unitary representation of $G$ and $f$ is an $L^{1}$ entire vector for $G$, then $w=\int_{G} f(g) U_{g} v d g$ is an entire vector for all $v$ in the representation space as follows from the identity

$$
\partial U(X) w=\int_{G_{0}}(-\partial L(X) f)(g) U_{g} v d g
$$

for all $X$ in the Lie algebra of $G$. We have then shown that every unitary representation of $G$ has a dense set of entire vectors. Such $G$ can be characterized simply as the not necessarily solvable class $R$ Lie groups due to the following lemma.

Lemma. $A$ Lie algebra $\mathcal{L}$ is class $R$ iff $\mathcal{L}$ is a semidirect product of a compact semisimple Lie algebra $\unlhd$ and a type $R$ solvable Lie algebra $\delta$.

Proof. Let $G$ be the c.s.c. Lie group corresponding to $\mathcal{L}$. If $\mathcal{L}$ is such a semidirect product, from the above we see that every unitary representation of $G$ must have dense sets of entire vectors. (II.1) then shows that $G$ must be type $R$. (Note that the proof of (II.1) nowhere assumed solvability of $G$.)

Conversely, if $\mathcal{L}$ is type $R$, let $\mathcal{L}=\beth+\delta$ be the Levi decomposition ( $\supset$ semisimple and $\delta$ solvable). Since $\delta$ is automatically type $R$, it suffices to show that $\beth$ is compact. Let $B$ be the Killing form for $\square$ and let $X \in \supset$. Since $\operatorname{tr}(\operatorname{ad} X$ ad $X)=B(X, X)$ is the sum of the squares of the eigenvalues of ad $X$ weighted according to multiplicity, $B(X, X) \leq 0$. Thus, since $B$ is nondegenerate, $B$ is negative definite and $\square$ is compact. Q.E.D.

Corollary (II.5). Corollary (II.4) is true with "solvable" omitted.

Acknowledgements. The author would like to thank his thesis advisor, Roe Goodman, for inspiring and guiding him in this work. The author's debt to Professor Goodman extends far beyond the present work. The author would also like to thank the NSF for supporting him while this work was being done. He would also like to thank the referee for his useful comments.

\section{REFERENCES}

1. L. Auslander and C. C. Moore, Unitary representations of solvable Lie groups, Mem. Amer. Math. Soc. No. 62 (1966). MR 34 \#7723.

2. R. Goodman, Analytic and entire vectors for representations of Lie groups, Trans. Amer. Math. Soc. 143 (1969), 55-76. MR 40 \#1537. 
3. R. Goodman, Complex Fourier analysis on nilpotent Lie groups, Trans. Amer. Math. Soc. 160 (1971), 373-391.

4. - Differential operators of infinite order on a Lie group. I, J. Math. Mech. 19 (1969/ 70), 879-894. MR 41 \#396.

5. A. Grothendieck, Espaces vectoriels topologiques, Sociedade de Mathematica de Sao Paulo, Sao Paulo, 1958.

6. G. Hochschild, The structure of Lie groups, Holden-Day, San Francisco, Calif., 1965. MR 34 \#7696.

7. R. T. Moore, Measurable, continuous and smooth vectors for semigroups and group representations, Mem. Amer. Math. Soc. No. 78 (1968). MR 37 \#4669.

8. E. Nelson, Analytic vectors, Ann. of Math. (2) 70 (1959), 572-615. MR 21 \#5901.

Department of Mathematics, Purdue University, Lafayette, Indiana 47907 